\title{
Local Computability for Ordinals
}

\author{
Johanna N.Y. Franklin ${ }^{1}$, Asher M. Kach ${ }^{2 \star}$, Russell Miller ${ }^{3 \star \star}$, and Reed \\ Solomon ${ }^{1}$ \\ 1196 Auditorium Road, Univ. of Connecticut, U-3009, Storrs, CT 06269-3009 U.S.A. \\ johanna.franklin@uconn.edu, http://www.math.uconn.edu/ franklin/ \\ david.solomon@uconn.edu, http://www.math.uconn.edu/ solomon/ \\ 2 University of Chicago, 5734 S. University Avenue, Chicago, IL 60637 U.S.A. \\ asher.kach@gmail.com, http://www.math.uchicago.edu/ kach/ \\ 3 Queens College of CUNY, 65-30 Kissena Blvd., Queens, NY 11367-1597 USA \\ and CUNY Graduate Center, 365 Fifth Avenue, New York, NY 10016 USA \\ Russell.Miller@qc.cuny.edu, http://qcpages.qc.cuny.edu/ rmiller
}

\begin{abstract}
We examine the extent to which well orders satisfy the properties of local computability, which measure how effectively the finite suborders of the ordinal can be presented. Known results prove that all computable ordinals are perfectly locally computable, whereas $\omega_{1}^{C K}$ and larger countable ordinals are not. We show that perfect local computability also fails for uncountable ordinals, and that ordinals $\alpha \geq \omega_{1}^{C K}$ are $\theta$-extensionally locally computable for all $\theta<\omega_{1}^{C K}$, but not when $\theta>\omega_{1}^{C K}$, nor when $\theta=\omega_{1}^{C K} \leq \alpha<\omega_{1}^{C K} \cdot \omega$.
\end{abstract}

Keywords: Computability theory, computable model theory, local computability, ordinal, recursion theory.

\section{Introduction}

Local computability represents an effort to give effective presentations of structures, such as the fields of real and complex numbers, which admit computation on their elements by simple algebraic algorithms and therefore, despite their uncountability, feel as though they ought to have computable presentations. Full definitions and much more analysis are given in [3-5], and we offer some basic definitions below. Local computability applies to linear orders as well as to fields and other structures, and the intention of this work is to investigate local computability for ordinals, the most ubiquitous linear orders in mathematical logic. We started with a particular eye on uncountable ordinals, but soon found large (noncomputable) countable ordinals to be of similar interest. For example,

* The second author was partially supported by a grant from the Packard Foundation through a Post-Doctoral Fellowship.

** The third author was partially supported by grant \# DMS - 1001306 from the National Science Foundation, by the Centre de Recerca Matemática, the Isaac Newton Institute, and the European Science Foundation, and by several PSC-CUNY grants from the Research Foundation of The City University of New York. 
can we use the ideas and terminology of local computability to draw distinctions between noncomputable countable ordinals and uncountable ordinals? Or between limit and successor ordinals? Or between cardinals and noncardinals? In this paper, we give almost complete negative answers to these questions. All noncomputable ordinals are $\alpha$-extentionally locally computable (defined below) for $\alpha<\omega_{1}^{C K}$ and are not $\beta$-extensionally locally computable for $\beta>\omega_{1}^{C K}$. If $\omega_{1}^{C K} \leq \gamma \leq \omega_{1}^{C K} \cdot \omega$, then $\gamma$ is not $\omega_{1}^{C K}$-extentionally locally computable, but it remains open whether there could be a larger ordinal which is $\omega_{1}^{C K}$-extentionally locally computable.

We now give the background definitions. In this context, one typically works with a fixed class of structures which is closed under taking finitely generated substructures. For example, one might consider the models of a $\forall$-axiomatizable theory $T$ or (as we do here) a nonaxiomatizable class of models such as the ordinals.

Definition 1. A simple cover of a structure $\mathcal{S}$ is a countable collection $\mathfrak{A}$ of models $\left\{\mathcal{A}_{i}\right\}_{i \in I}$ of $T$, each generated by a finite tuple $\boldsymbol{a}_{i}$, such that every finitely generated substructure of $\mathcal{S}$ is isomorphic to some $\mathcal{A}_{i}$ and every $\mathcal{A}_{i}$ embeds into $\mathcal{S}$.

A simple cover is computable if every $\mathcal{A}_{i} \in \mathfrak{A}$ is a computable structure with domain an initial segment of $\omega$.

A simple cover is uniformly computable if the sequence $\left\{\left(\mathcal{A}_{i}, \boldsymbol{a}_{i}\right)\right\}_{i \in I}$ can be given uniformly computably, including a strong index for each $\boldsymbol{a}_{i}$.

Definition 2. A cover of $\mathcal{S}$ consists of a simple cover $\mathfrak{A}$ of $\mathcal{S}$ along with sets $I_{i j}^{\mathfrak{A}}$ (for all $\mathcal{A}_{i}, \mathcal{A}_{j} \in \mathfrak{A}$ ) of injective homomorphisms $f: \mathcal{A}_{i} \hookrightarrow \mathcal{A}_{j}$ satisfying:

- For all finitely generated substructures $\mathcal{B} \subseteq \mathcal{C} \subseteq \mathcal{S}$, there exists $i, j \in \omega$, $f \in I_{i j}^{\mathfrak{A}}$, and $\beta: \mathcal{A}_{i} \cong \mathcal{B}$ and $\gamma: \mathcal{A}_{j} \cong \mathcal{C}$ with $\beta=\gamma \circ f$.

- For all $k$ and $m$ and $g \in I_{k, m}^{\mathfrak{A}}$, there exist finitely generated substrutures $\mathcal{D} \subseteq \mathcal{E} \subseteq \mathcal{S}$ and isomorphisms $\delta: A_{k} \cong \mathcal{D}$ and $\epsilon: A_{m} \cong \mathcal{E}$ with $\delta=\epsilon \circ \mathrm{g}$.

- The Amalgamation Property: for every $i, j, k$ and all maps $f \in I_{i j}^{\mathfrak{A}}$ and $g \in I_{i k}^{\mathfrak{A}}$, there exists $m$ and maps $h \in I_{j m}^{\mathfrak{A}}$ and $p \in I_{k m}^{\mathfrak{A}}$ with $p \circ g=h \circ f$.

We often refer to the elements of $\mathfrak{A}$ as the objects of the cover and the elements of the $I_{i j}^{\mathfrak{A}}$ as the maps of the cover.

$A$ cover is computable if $\mathfrak{A}$ is a uniformly computable simple cover of $\mathcal{S}$ and there exists a c.e. set $W$ such that, for all $i, j \in \omega$,

$$
I_{i j}^{\mathfrak{A}}=\left\{\varphi_{e}\left\lceil\mathcal{A}_{i}:\langle i, j, e\rangle \in W\right\} .\right.
$$

The structure $\mathcal{S}$ is locally computable if it has a uniformly computable cover.

In past articles on local computability, the Amalgamation Property has not always been included in the definition of a cover. Therefore, in the interest of clarity, we will occasionally remind the reader that we are working in the context of this property.

Definition 3. Let $\mathfrak{A}$ be a cover of a structure $\mathcal{S}$. An $\mathcal{A}_{i} \in \mathfrak{A}$ matches a substructure $\mathcal{B} \subseteq \mathcal{S}$ extensionally if there is an isomorphism $\beta: \mathcal{A}_{i} \cong \mathcal{B}$ satisfying: 
- For every finitely generated $\mathcal{C}$ with $\mathcal{B} \subseteq \mathcal{C} \subseteq \mathcal{S}$, there exists $j \in \omega, f \in I_{i j}^{\mathfrak{A}}$, and $\gamma: \mathcal{A}_{j} \cong \mathcal{C}$ with $\beta=\gamma \circ f$.

- For every $m \in \omega$ and $g \in I_{i, m}^{\mathfrak{A}}$, there exists an $\mathcal{E} \subseteq \mathcal{S}$ and $\epsilon: \mathcal{A}_{m} \cong \mathcal{E}$ with $\mathcal{B} \subseteq \mathcal{E}$ and $\beta=\epsilon \circ g$.

The map $\beta$ is termed an extensional match between $\mathcal{A}_{i}$ and $\mathcal{B}$.

Definition 4. Let $\mathfrak{A}$ be a cover of a structure $\mathcal{S}$. Every isomorphism $\beta: \mathcal{A}_{i} \cong \mathcal{B}$, where $\mathcal{B} \subseteq \mathcal{S}$ is a finitely generated substructure, is 0 -extensional.

For an ordinal $\theta>0$, an isomorphism $\beta: \mathcal{A}_{i} \cong \mathcal{B}$ is $\theta$-extensional if:

- For every finitely generated $\mathcal{C}$ with $\mathcal{B} \subseteq \mathcal{C} \subseteq \mathcal{S}$ and every ordinal $\zeta<\theta$, there exists $j \in \omega, f \in I_{i j}^{\mathfrak{A}}$, and a $\zeta$-extensional $\gamma: \mathcal{A}_{j} \cong \mathcal{C}$ with $\beta=\gamma \circ f$.

- For every $m \in \omega, g \in I_{i, m}^{\mathfrak{A}}$, and ordinal $\zeta<\theta$, there exists $\mathcal{E} \subseteq \mathcal{S}$ and $\zeta$-extensional $\epsilon: \mathcal{A}_{m} \cong \mathcal{E}$ with $\mathcal{B} \subseteq \mathcal{E}$ and $\beta=\epsilon \circ g$.

$A$ uniformly computable cover $\mathfrak{A}$ of $\mathcal{S}$ is $\theta$-extensional if for every $\mathcal{A}_{i} \in \mathfrak{A}$ there is a $\theta$-extensional isomorphism $\beta: \mathcal{A}_{i} \cong \mathcal{B}$ to some finitely generated substructure $\mathcal{B} \subseteq \mathcal{S}$ and for every finitely generated substructure $\mathcal{E} \subseteq \mathcal{S}$ there is a $\theta$-extensional isomorphism $\epsilon: \mathcal{A}_{j} \cong \mathcal{E}$ from some $\mathcal{A}_{j} \in \mathfrak{A}$.

If such a uniformly computable cover exists, we say that $\mathcal{S}$ is $\theta$-extensionally locally computable or, more simply, $\theta$-extensional.

Definition 5. Let $\mathfrak{A}$ be a uniformly computable cover for a structure $\mathcal{S}$. A set $M$ is a correspondence system for $\mathfrak{A}$ and $\mathcal{S}$ if it satisfies:

- Each element of $M$ is an embedding of an $\mathcal{A}_{i}$ into $\mathcal{S}$.

- For every $\mathcal{A}_{i} \in \mathfrak{A}$, there exists a $\beta \in M$ with domain $\mathcal{A}_{i}$.

- For every finitely generated substructure $\mathcal{B} \subseteq \mathcal{S}$, there exists a $\beta \in M$ with range $\mathcal{B}$.

- For every $\mathcal{A}_{i} \in \mathfrak{A}$, every $\beta \in M$ with domain $\mathcal{A}_{i}$, and every finitely generated substructure $\mathcal{C} \subseteq \mathcal{S}$ with $\beta\left(\mathcal{A}_{i}\right) \subseteq \mathcal{C}$, there exists $\mathcal{A}_{j} \in \mathfrak{A}, \gamma \in M$ with domain $\mathcal{A}_{j}$ and image $\mathcal{C}$, and $f \in I_{i j}^{\mathfrak{A}}$ with $\beta=\gamma \circ f$.

- For every $\mathcal{A}_{i} \in \mathfrak{A}$, every $\beta \in M$ with domain $\mathcal{A}_{i}$, and every $\mathcal{A}_{m} \in \mathfrak{A}$ and every $g \in I_{i, m}^{\mathfrak{A}}$, there exists $\epsilon \in M$ with domain $\mathcal{A}_{m}$ with $\beta=\epsilon \circ \mathrm{g}$.

If $\mathcal{S}$ has a uniformly computable cover $\mathfrak{A}$ with a correspondence system $M$, then we say $\mathcal{S}$ is $\infty$-extensionally locally computable.

However, the definition applies perfectly well to countable structures, and Miller proved the following connection between local computability and computable presentability for countable structures (see $[5,4]$ ).

Theorem 1. Let $\mathcal{S}$ be a countable structure. $\mathcal{S}$ is isomorphic to a computable structure if and only if $\mathcal{S}$ has an $\infty$-extensional computable cover (with the AP).

This equivalence helped to establish $\infty$-extensionality as the ultimate goal, when one desires to prove that a particular structure of arbitrary cardinality is "nicely presentable." It also justifies our decision to consider only covers with the AP. 


\section{Failures of Extensionality for Ordinals}

We now show that having sufficiently high ordinal levels of extensionality is sufficient for $\infty$-extensional local computability. We work in $\mathcal{L}_{\omega_{1}, \omega}$ allowing countable conjunctions and disjunctions, and we use the standard notation $\Sigma_{\alpha}$ for $\alpha<\omega_{1}$ to calibrate the complexity of our formulas. (See [1] for additional background on this logic.)

Lemma 1. Let $\mathfrak{A}$ be a cover of a structure $\mathcal{S}$. Suppose $\mathcal{A}_{i} \in \mathfrak{A}$ and $\psi: \mathcal{A}_{i} \rightarrow \mathcal{C}$ is a $\theta$-extensional map onto a substructure $\mathcal{C}$ of $\mathcal{S}$, and let $h$ be an automorphism of $\mathcal{S}$. Then $h \circ \psi$ is also $\theta$-extensional.

Proof. We induct on $\theta$. For $\theta=0$, if $\psi$ is 0 -extensional, it is an injective homomorphism, and therefore so is $h \circ \psi$. Thus $h \circ \psi$ is 0 -extensional.

For $\theta>0$, if $f \in I_{i j}^{\mathfrak{A}}$ lifts to an inclusion $\mathcal{C} \subseteq \mathcal{D}$ via $\psi$ and a $\zeta$-extensional map $\varphi$ (for any $\zeta<\theta$ ), then $f$ also lifts to the inclusion $h(\mathcal{C}) \subseteq h(\mathcal{D})$ via $h \circ \psi$ and the map $h \circ \varphi$. By induction on $\theta$, the map $h \circ \varphi$ is also $\zeta$-extensional. It follows that $h \circ \psi$ is $\theta$-extensional.

Lemma 2. Suppose that $\mathfrak{A}$ is a computable cover of a structure $\mathcal{S}$, and that $\boldsymbol{a}$ is an $n$-tuple from an object $\mathcal{A}_{i} \in \mathfrak{A}$. If $\varphi$ and $\psi$ are both $\theta$-extensional maps from $\mathcal{A}_{i}$ into $\mathcal{S}$, then the tuples $\varphi(\boldsymbol{a})$ and $\psi(\boldsymbol{a})$ satisfy exactly the same $\Sigma_{\theta}$ formulas in $\mathcal{S}$.

We will sometimes refer to the set of these $\Sigma_{\theta}$ formulas as the $\Sigma_{\theta}$-theory of $\boldsymbol{a}_{i}$ in $\mathfrak{A}$, and will speak of $\boldsymbol{a}_{i}$ satisfying various formulas in $\mathfrak{A}$. The lemma can be seen as saying that this notion is well-defined: in the theory of the cover $\mathfrak{A}, \boldsymbol{a}_{i}$ satisfies exactly those $\Sigma_{\theta}$ formulas that its image, under an arbitrary $\theta$ extensional map, satisfies in $\mathcal{S}$. (Alternatively, one can define the $\Sigma_{\theta}$-theory of $\boldsymbol{a}_{i}$ in $\mathfrak{A}$ by using $\exists$-quantifiers to refer to the existence of embeddings $f \in I^{\mathfrak{A}}$ from $\mathcal{A}_{i}$ into other objects $\mathcal{A}_{j}$ of $\mathfrak{A}$, such that $\left(\mathcal{A}_{j}, f\left(\boldsymbol{a}_{i}\right)\right)$ satisfies the formula inside the $\exists$-quantifier. This is natural, and is equivalent to the above definition.)

Proof. For $\theta=0$, this follows from $\varphi$ and $\psi$ both being 0 -extensional, i.e., being embeddings of $\mathcal{A}_{i}$ into $\mathcal{S}$. For $\theta>0$, suppose

$$
\mathcal{S} \models \bigvee_{k \in \omega}(\exists y)\left[P_{k}(\psi(\boldsymbol{a}), y)\right]
$$

where each $P_{k}$ is a $\Pi_{\zeta_{k}}$-formula with $\zeta_{k}<\theta$. Fix $k \in \omega$ and $y \in \mathcal{S}$ such that $\mathcal{S} \models P_{k}(\psi(\boldsymbol{a}), y)$. The inclusion range $(\psi) \subseteq$ range $(\psi) \cup\{y\}$ in $\mathcal{S}$ must be the lift of some $f$ in some $I_{i j}^{\mathfrak{A}}$, via $\psi$ and some $\zeta_{k}$-extensional $\psi^{\prime}: \mathcal{A}_{j} \rightarrow \operatorname{range}(\psi) \cup\{y\}$. But this $f$ must also lift to an inclusion range $(\varphi) \subseteq \mathcal{D}$ in $\mathcal{S}$ via $\varphi$ and some $\zeta_{k^{-}}$ extensional $\varphi^{\prime}$. By induction, the $\Pi_{\zeta_{k}}$ formula $P_{k}(\boldsymbol{x}, y)$, being known to hold of $\left(\psi^{\prime}(f(\boldsymbol{a}), y)=(\psi(\boldsymbol{a}), y)\right.$, must also hold of $\left(\varphi^{\prime}(f(\boldsymbol{a})), z\right)=(\varphi(\boldsymbol{a}), z)$, where $z:=$ $\varphi^{\prime}\left(\psi^{\prime-1}(y)\right)$. Thus $\varphi(\boldsymbol{a})$ also satisfies $\bigvee_{k}(\exists y)\left[P_{k}(\boldsymbol{x}, y)\right]$. Finally, by a symmetric argument, if $\varphi(\boldsymbol{a})$ satisfies this $\Sigma_{\theta}$ formula, then so does $\psi(\boldsymbol{a})$. 
Several definitions of Scott rank exist in the literature (see [1]). For our purposes, only the following property of the Scott rank (and not the exact definition) matters: whenever $\mathcal{S}$ is a countable structure of Scott $\operatorname{rank} \zeta$ and $\boldsymbol{x}$ and $\boldsymbol{y}$ are $n$-tuples of elements from $\mathcal{S}$ (for any $n$ ) which satisfy exactly the same $\Pi_{\zeta}$ formulas in $n$ variables, there must exist an automorphism of $\mathcal{S}$ mapping each $x_{i}$ to the corresponding $y_{i}$.

Lemma 3. Fix ordinals $\theta$ and $\zeta$ with $\theta>\zeta$. Let $\mathfrak{A}$ be a $\theta$-extensional cover of a countable structure $\mathcal{S}$ with Scott rank $\zeta$. Suppose $\mathcal{A}_{i} \in \mathfrak{A}$ and $\psi: \mathcal{A}_{i} \rightarrow \mathcal{S}$ is a $\zeta$-extensional map. Then $\psi$ is also $\theta$-extensional.

Proof. Since $\mathfrak{A}$ is a $\theta$-extensional cover, we know that $\mathcal{A}_{i}$ is the domain of some $\theta$-extensional map $\varphi: \mathcal{A}_{i} \rightarrow \mathcal{S}$. Let $\boldsymbol{a}$ be a finite tuple generating $\mathcal{A}_{i}$. Then by Lemma 2 , the tuples $\varphi(\boldsymbol{a})$ and $\psi(\boldsymbol{a})$ satisfy exactly the same $\Pi_{\zeta}$-formulas in $\mathcal{S}$. Since $\mathcal{S}$ has Scott rank $\zeta$, there must be an automorphism $h$ of $\mathcal{S}$ mapping $\varphi(\boldsymbol{a})$ onto $\psi(\boldsymbol{a})$. But then $h \circ \varphi=\psi$ since $\boldsymbol{a}$ generates $\mathcal{A}_{i}$, and so by Lemma 1 , the map $\psi$ is also $\theta$-extensional.

Proposition 1. For a countable structure $\mathcal{S}$, the following are equivalent.

1. The structure $\mathcal{S}$ is computably presentable.

2. The structure $\mathcal{S}$ is perfectly locally computable (as defined in [5]).

3. The structure $\mathcal{S}$ has an $\infty$-extensional computable cover with the Amalgamation Property.

4. There is an ordinal $\theta$ strictly greater than the $S$ cott rank of $\mathcal{S}$, such that $\mathcal{S}$ has a $\theta$-extensional computable cover with the Amalgamation Property.

Proof. The equivalence of (1), (2), and (3) is shown in [4, Thm 6.3]; some of it was originally proven by Miller and Mulcahey in [5]. Since (3) $\Longrightarrow(4)$ is trivial, we need only show that $(4) \Longrightarrow(3)$. Fix a $\theta$-extensional computable cover $\mathfrak{A}$ of $\mathcal{S}$. We claim that the set $M$ of all $\theta$-extensional maps $\psi$ of objects $\mathcal{A}_{i}$ into $\mathcal{S}$ must be a correspondence system. Clearly every $\mathcal{A}_{i}$ is the domain of such a map and every finitely generated $\mathcal{D} \subseteq \mathcal{S}$ is the image of such a map. Moreover, for any $\psi \in M$, say with domain $\mathcal{A}_{i}$, and every $f \in I_{i j}^{\mathfrak{A}}$, we can lift $f$ to an inclusion via $\psi$ and a $\zeta$-extensional $\varphi$ (since $\zeta<\theta$ ), and by Lemma $3, \varphi$ is also in $M$. Likewise, every inclusion of range $(\psi)$ is the lift of some $f \in I_{i j}^{\mathfrak{A}}$, for some $j$, via some $\zeta$-extensional $\varphi$, and again, by Lemma 3 , this $\varphi$ actually lies in $M$.

Corollary 1. For every $\theta>\omega_{1}^{C K}$, the ordinal $\omega_{1}^{C K}$ is not $\theta$-extensionally locally computable.

Proof. The Scott rank of the ordinal $\omega_{1}^{C K}$ (as a linear order) is exactly $\omega_{1}^{C K}$. Hence, as a countable structure with no computable presentation, $\omega_{1}^{C K}$ cannot be $\theta$-extensionally locally computable for any $\theta>\omega_{1}^{C K}$, by Proposition 1 .

In Proposition 2 and Theorem 3, we strengthen this corollary to cover some of the case $\theta=\omega_{1}^{C K}$. The situation for $\theta<\omega_{1}^{C K}$ will be handled in Theorem 4, and the rest of the case $\theta>\omega_{1}^{C K}$ in Theorem 2 . 
Lemma 4 (Folklore; see e.g. [1]). For each finite sequence of ordinal $\alpha_{0}<$ $\cdots<\alpha_{k}<\omega_{1}^{C K}$, there is a computable infinitary formula $\lambda\left(x_{0}, \ldots, x_{k}\right)$ (in the language of linear orders) such that for every ordinal $\gamma, \gamma \models \lambda\left(\beta_{0}, \ldots, \beta_{k}\right)$ if and only if $\beta_{i}=\alpha_{i}$ for each $i \leq k$.

Proposition 2. There is no $\omega_{1}^{C K}$-extensional computable cover (with AP) of the ordinal $\omega_{1}^{C K}$ itself.

Proof. Suppose $\mathfrak{A}$ were such a cover. By Theorem 1, if there were a correspondence system $M$ for $\mathfrak{A}$ and $\omega_{1}^{C K}$, then there would be a computable copy of $\omega_{1}^{C K}$, yielding a contradiction. Our goal is to define such a correspondence system $M$.

For any $\mathcal{A}_{i} \in \mathfrak{A}$ with $\mathcal{A}_{i}=\mathbf{a}$, let $\psi$ and $\psi^{\prime}$ be $\omega_{1}^{C K}$-extensional maps from $\mathcal{A}_{i}$ into $\omega_{1}^{C K}$. The tuples $\psi(\mathbf{a})$ and $\psi^{\prime}(\mathbf{a})$ satisfy the same $\Pi_{\omega_{1}^{C K}}$ formulas in $\omega_{1}^{C K}$. By Lemma 4 , this forces $\psi$ and $\psi^{\prime}$ to agree on $\mathbf{a}$, and hence on $\mathcal{A}_{i}$. Thus, every $\mathcal{A}_{i} \in \mathfrak{A}$ is the domain of exactly one $\omega_{1}^{C K}$-extensional map $\psi_{i}$ into $\omega_{1}^{C K}$. In fact, for every $\mathcal{A}_{i}$, there is a $\delta_{i}<\omega_{1}^{C K}$ such that $\psi_{i}$ is the unique $\delta_{i}$-extensional map of $\mathcal{A}_{i}$ into $\omega_{1}^{C K}$. (We assume $\delta_{i}$ is least with this property.)

Let $M$ be the collection of all $\omega_{1}^{C K}$-extensional maps $\psi_{i}$. We claim $M$ is a correspondence system. The first three properties of a correspondence system follow immediately from the fact that $\mathfrak{A}$ is an $\omega_{1}^{C K}$-extensional computable cover.

To verify the fourth property, fix $\mathcal{A}_{i} \in \mathfrak{A}$ and $\beta \in M$ with domain $\mathcal{A}_{i}$. Since $\beta$ is $\omega_{1}^{C K}$-extensional, $\beta=\psi_{i}$. Fix a finite $\mathcal{C}$ such that $\psi_{i}\left(\mathcal{A}_{i}\right) \subseteq \mathcal{C} \subseteq \omega_{1}^{C K}$. By Lemma 4 , let $\theta<\omega_{1}^{C K}$ be such that $\mathcal{C}$ is defined by a $\Sigma_{\theta}$ formula in $\omega_{1}^{C K}$. Using the fact that $\mathfrak{A}$ is an $\omega_{1}^{C K}$-extensional cover, fix $\mathcal{A}_{j}, f \in I_{i j}^{\mathfrak{A}}$ and an $\theta$-extensional map $\gamma$ such that $\beta=\psi_{i}=\gamma \circ f$. Since $\mathcal{C}$ is defined by a $\Sigma_{\theta}$ formula, it follows that $\delta_{j} \leq \theta$ and hence $\gamma=\psi_{j}$. Therefore, $\gamma$ is $\omega_{1}^{C K}$-extensional and hence $\gamma \in M$ as required.

The fifth property follows by an similar argument which we leave to the reader.

Theorem 2. If $\alpha>\omega_{1}^{C K}$, then $\alpha$ has no $\left(\omega_{1}^{C K}+1\right)$-extensional computable cover with the Amalgamation Property. Indeed, no computable cover of such an $\alpha$ can have any object with an $\left(\omega_{1}^{C K}+1\right)$-extensional map into $\alpha$ whose image contains $\omega_{1}^{C K}$.

Proof. We show that from such a cover $\mathfrak{A}$, we could construct a computable presentation $\mathcal{S}$ of $\omega_{1}^{C K}$. Let $\mathcal{A}_{i_{0}}$ be the object with an $\left(\omega_{1}^{C K}+1\right)$-extensional map $\varphi_{0}$ such that $\varphi_{0}\left(x_{0}\right)=\omega_{1}^{C K}$ for some $x_{0} \in \mathcal{A}_{i_{0}}$ (hereafter fixed). The argument in a nutshell is that we can watch for embeddings $g \in I^{\mathfrak{A}}$ mapping $\mathcal{A}_{i_{0}}$ into other objects $\mathcal{A}_{j}$ of $\mathfrak{A}$. When we find such a $g$, it must lift to an inclusion in $\alpha$ via $\varphi_{0}$ and some $\omega_{1}^{C K}$-extensional $\varphi_{1}$, and so every element $y<g\left(x_{0}\right)$ in $\mathcal{A}_{j}$ is forced to map to some ordinal $<\varphi_{1}\left(g\left(x_{0}\right)\right)=\omega_{1}^{C K}$ in $\alpha$. Since the map is $\omega_{1}^{C K_{-}}$ extensional, Lemma 2 shows that in the theory of $\mathfrak{A}, y$ satisfies some identifying formula from Lemma 4 . We then use the AP to amalgamate $\mathcal{A}_{j}$ together with the portion of $\mathcal{S}$ already built, and either we see that $y$ maps to some element already in $\mathcal{S}$, or else we add a new element to $\mathcal{S}$ to correspond to this $y$. Since 
every ordinal $<\omega_{1}^{C K}$ corresponds to some such $y$ in some such $\mathcal{A}_{j}$, the $\mathcal{S}$ built this way is actually a copy of $\omega_{1}^{C K}$.

We start building $\mathcal{S}$ by setting $\mathcal{B}_{0}=\left\{y \in \mathcal{A}_{i_{0}}: y<x\right\}$, letting $\mathcal{S}_{0}$ be the (possibly empty) linear order $\left\{0,1, \ldots,\left|\mathcal{B}_{0}\right|-1\right\}$ under $<$, and defining $p_{0}: \mathcal{S}_{0} \rightarrow$ $\mathcal{B}_{0}$ to be an order-isomorphism.

At stage $s+1$, we begin with a finite order $\mathcal{S}_{s}$ and with some sequence of objects and embeddings from $\mathfrak{A}$, given effectively:

$$
\mathcal{A}_{i_{0}} \hookrightarrow \mathcal{A}_{i_{1}} \hookrightarrow \cdots \hookrightarrow \mathcal{A}_{i_{s}}
$$

where each embedding $f_{t}: \mathcal{A}_{i_{t}} \hookrightarrow \mathcal{A}_{i_{t+1}}$ lies in $I_{i_{t} i_{t+1}}^{\mathfrak{A}}$. By induction, we know an isomorphism $p_{s}$ from $\mathcal{S}_{s}$ onto a suborder $\mathcal{B}_{s}$ of $\mathcal{A}_{i_{s}}$, with every element of $\mathcal{B}_{s}$ below the element $x_{s}=f_{s-1}\left(x_{s-1}\right)$ in $\mathcal{A}_{i_{s}}$, which is the image of $x_{0}$ under $\left(f_{s-1} \circ \cdots \circ f_{0}\right)$.

We now search through $I^{\mathfrak{A}}$ for the first map $g_{0, s}$ such that:

$-g_{0, s} \in I_{i_{0}, j_{0, s}}^{\mathfrak{A}}$ for some $j_{0, s} ;$ and

- $\mathcal{A}_{j_{0, s}}$ contains exactly one $y<g_{0, s}(x)$ which is not in $\operatorname{range}\left(g_{0, s}\right)$; and

- $g_{0, s}$ has not been considered at any previous stage.

Such a $g_{0, s}$ must exist, since there are infinitely many elements of $\alpha$ lying below $\omega_{1}^{C K}$ satisfying distinct computable infinitary formulas in $\alpha$. Once we find the least one, we fix it and search for amalgamations: first $j_{1, s} \in \omega$ and $g_{1, s}, h_{0, s} \in I^{\mathfrak{A}}$, then $j_{2, s} \in \omega$ and $g_{2, s}, h_{1, s} \in I^{\mathfrak{A}}$, etc., as shown here:

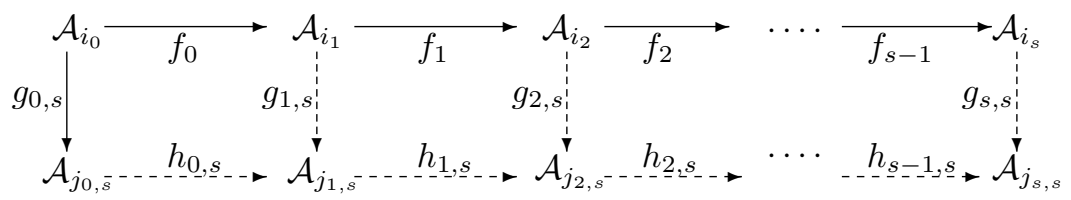

We define $i_{s+1}=j_{s, s}$ and $f_{s}=g_{s, s}$, thus adding $\mathcal{A}_{j_{s, s}}$ to the sequence $\mathcal{A}_{i_{0}}, \mathcal{A}_{i_{1}}, \ldots$ previously built. If the image of $\mathcal{B}_{s}$ under $f_{s}$ already contains the element $y_{s+1}=\left(h_{s-1, s} \circ h_{s-2, s} \circ \cdots \circ h_{0, s}\right)\left(g_{0, s}((y))\right.$, then we set $\mathcal{S}_{s+1}=\mathcal{S}_{s}$ and $p_{s+1}=f_{s} \circ p_{s}$. If not, then we extend $\mathcal{S}_{s}$ to a larger order $\mathcal{S}_{s+1}$ by adding one new element $z_{s+1}$ to $\mathcal{S}_{s}$, with $p_{s+1}\left(z_{s+1}\right)=y_{s+1}$ and $p_{s+1}=f_{s} \circ p_{s}$ on the rest of $\mathcal{S}_{s+1}$. The order on $\mathcal{S}_{s+1}$ is defined so that $p_{s+1}$ remains an order isomorphism from $\mathcal{S}_{s+1}$ into the suborder $\mathcal{B}_{s+1}=\mathcal{B}_{s} \cup\left\{y_{s+1}\right\}$ of $\mathcal{A}_{i_{s+1}}$; clearly this is compatible with the order on $\mathcal{S}_{s}$, and it justifies the inductive hypothesis at the next stage.

This is the entire construction, building the computable linear order $\mathcal{S}=$ $\cup_{s} \mathcal{S}_{s}$. We now present the (non-effective) inductive argument that $\mathcal{S} \cong \omega_{1}^{C K}$, which proceeds through the same stages just described. At stage 0, of course we have an $\left(\omega_{1}^{C K}+1\right)$-extensional map $\varphi_{0}: \mathcal{A}_{i_{0}} \rightarrow \alpha$, and we define $\psi_{0}=\varphi_{0} \circ p_{0}$, embedding $\mathcal{S}_{0}$ isomorphically into $\omega_{1}^{C K}$ within $\alpha$ (since $\varphi_{0}\left(x_{0}\right)=\omega_{1}^{C K}$, and $p_{0}$ maps all elements of $\mathcal{S}_{0}$ to elements below $x_{0}$ in $\mathcal{A}_{i_{0}}$ ).

Now at stage $s+1$ we chose an embedding $g_{0, s}: \mathcal{A}_{i_{0}} \hookrightarrow \mathcal{A}_{j_{0, s}}$ from $I^{\mathfrak{A}}$. Since $\varphi_{0}$ is $\left(\omega_{1}^{C K}+1\right)$-extensional, this $g_{0, s}$ lifts to an inclusion range $\left(\varphi_{0}\right) \subseteq \mathcal{C}$, for some finite $\mathcal{C} \subseteq \alpha$, via $\varphi_{0}$ and some $\omega_{1}^{C K}$-extensional map $\varphi_{1, s}$ sending $\mathcal{A}_{j_{0, s}}$ 
onto $\mathcal{C}$. By Lemma 4 , then, the unique $y<g_{0, s}(x)$ in $\left(\mathcal{A}_{j_{0, s}}-\right.$ range $\left.\left(g_{0, s}\right)\right)$ must satisfy (in the theory of the cover $\mathfrak{A}$ ) some computable infinitary formula which uniquely identifies one computable ordinal. Fix some $\theta_{s}$ with $\theta_{s-1}<\theta_{s}<\omega_{1}^{C K}$ large enough that this formula is $\Sigma_{\theta_{s}}$.

Now we proceed along the diagram above. Each object $\mathcal{A}_{i_{t}}$ is the domain of some $\left(\theta_{s}\right)$-extensional map into $\alpha$, as is each object $\mathcal{A}_{j_{t s}}$, such that these maps are all compatible with $\varphi_{0}$. To see this, take $\left(\theta_{s}+s\right)$-extensional maps with domains $\mathcal{A}_{i_{1}}$ and $\mathcal{A}_{j_{0, s}}$, using $\left(\omega_{1}^{C K}+1\right)$-extensionality of $\varphi_{0}$; then $\left(\theta_{s}+s-1\right)$ extensional maps with domains $\mathcal{A}_{i_{2}}$ and $\mathcal{A}_{j_{1, s}}$, etc. Notice that for an $\mathcal{A}_{j_{t, s}}$ with $t>0$, we may have several different such maps, depending on the path one takes through the diagram. However, every element $y$ from any $\mathcal{B}_{t}$ within $\mathcal{A}_{i_{t}}$ satisfies a $\Sigma_{\theta_{s}}$ formula from Lemma 4 , and therefore has a unique possible image in $\alpha$ under these $\theta_{s}$-extensional maps: there is only one element in $\alpha$ satisfying that formula. Moreover, for such a $y \in \mathcal{B}_{t}$, the same holds of the element $g_{t, s}(y)$ of $\mathcal{A}_{j_{t, s}}$ under all $\theta_{s}$-extensional maps from $\mathcal{A}_{j_{t, s}}$ into $\alpha$. So all of these maps agree on all elements of $\mathcal{B}_{s}$ and on their images in $\mathcal{A}_{j_{s, s}}$. Indeed, this remains true even when we allow $s$ to vary: $\theta_{s}$ will be larger for larger $s$, and $\mathcal{A}_{j_{t, s}}$ may be distinct from $\mathcal{A}_{j_{t, s+1}}$, but each element of any $\mathcal{B}_{t}$ within each $\mathcal{A}_{i_{t}}$ in the diagram at stage $s+1$ is mapped to the same element of $\alpha$ by all these maps at this and all subsequent stages. So, to define $\psi_{s}(z)$ for $z \in \mathcal{S}_{s}$, we just map $z$ into $\mathcal{B}_{s}$ using $p_{s}$, and then send $p_{s}(z)$ to its image in $\alpha$ under any one of these $\theta_{s}$-extensional maps. This defines $\psi_{s}$ unambiguously on $\mathcal{S}_{s}$, and each $\psi_{s}$ is compatible with $\psi_{s+1}$, because $p_{s+1}$ restricts to $p_{s}$ and because we noted above that the image of an element of $\mathcal{B}_{s}$ below the image of $x$ has only one possible image in $\alpha$ under these (sufficiently extensional) maps. So it is clear that this $\psi=\cup_{s} \psi_{s}$ is an embedding of $\mathcal{S}$ into $\omega_{1}^{C K}$ within $\alpha$. Finally, for each element $\gamma \notin \operatorname{range}\left(\varphi_{0}\right)$ of the linear order $\omega_{1}^{C K}$, there is some $j_{0}$ and some map $g_{0}: \mathcal{A}_{i_{0}} \rightarrow \mathcal{A}_{j_{0}}$ which lifts to the inclusion range $\left(\varphi_{0}\right) \subseteq \operatorname{range}\left(\varphi_{0}\right) \cup\{\gamma\}$, and at some stage $s$ this $j_{0}$ and this $g_{0}$ will be chosen as $j_{0, s}$ and $g_{0, s}$. At that stage, $\gamma$ will become the $\psi_{s}$-image of some element of $\mathcal{S}_{s}$, and so the embedding $\psi$ actually maps $\mathcal{S}$ onto $\omega_{1}^{C K}$. Thus $\mathcal{S}$ is a computable presentation of $\omega_{1}^{C K}$, which is impossible.

It remains to decide whether an $\alpha \geq \omega_{1}^{C K}$ could have an $\omega_{1}^{C K}$-extensional computable cover. In the initial cases, we can answer this.

Theorem 3. If $\omega_{1}^{C K} \leq \alpha<\omega_{1}^{C K} \cdot \omega$, then $\alpha$ has no $\omega_{1}^{C K}$-extensional computable cover with $A P$.

Proof. We sketch the proof, which mixes the techniques used for Proposition 2 and Theorem 2. Now one fixes some $i_{0}$ for which $\mathcal{A}_{i_{0}}$ is the domain of an $\omega_{1}^{C K} K_{-}$ extensional map $\varphi_{0}$ onto the finite set $\alpha \cap\left\{\omega_{1}^{C K} \cdot(n+1): n \in \omega\right\}$. Consider any $j$ and any $g \in I_{i_{0} j}^{\mathfrak{A}}$. Now for every $\theta<\omega_{1}^{C K}$, every $g(x)$ maps to $\varphi_{0}(x)$ by some $\theta$-extensional map, and so each $g(x)$ satisfies a $\Sigma_{\theta}$-formula in $\mathfrak{A}$ stating that in the Cantor normal form of $g(x)$, every $\omega^{\zeta}$ with $\zeta<\theta$ has coefficient 0 . Since this holds for all $\theta<\omega_{1}^{C K}$, each $\omega_{1}^{C K}$-extensional map $\psi$ with domain $\mathcal{A}_{j}$ must send each of these $g(x)$ to a nonzero multiple of $\omega_{1}^{C K}$ in $\alpha$. If follows that each element $y \in \mathcal{A}_{j}$ with $y<\min (\operatorname{range}(g))$ has $\psi(y)<\omega_{1}^{C K}$ in $\alpha$. By Lemma 4 , each such $y$ 
satisfies a $\Sigma_{\theta}$ formula in $\mathfrak{A}$ which, in all ordinals, can only be satisfied by $\psi(y)$. This allows us to run the same construction that we did in Theorem 2, going systematically through maps $g \in I^{\mathfrak{A}}$ from $\mathcal{A}_{i_{0}}$ into any $\mathcal{A}_{j}$ in such a way that $\min (\operatorname{range}(g)) \neq \min \left(\mathcal{A}_{j}\right)$ and amalgamating those maps into the construction to get a computable presentation of $\omega_{1}^{C K}$, which is impossible.

\section{$3 \quad$ Extensionality for Ordinals Beyond $\omega_{1}^{C K}$}

Theorem 4. For each computable ordinal $\theta$, every ordinal $\alpha$ has a $\theta$-extensional computable cover.

The full proof is too long to present in this context, but we can provide a number of details. We state the key lemmas (in terms of the fixed computable ordinal $\theta$ ), present the proof of Theorem 4 assuming these lemmas, and end with a sketch of the proofs of the lemmas.

Lemma 5. If linear orders $\mathcal{S}_{0}$ and $\mathcal{S}_{1}$ each have $\theta$-extensional computable covers, then so does their sum $\mathcal{S}_{0}+\mathcal{S}_{1}$.

Lemma 6. Each ordinal multiple of $\omega^{\theta}$ of the form $\omega^{\theta} \cdot \beta$ (with $\beta \geq \omega$ ) has a $\theta$-extensional computable cover.

To prove Theorem 4 , notice that every computable ordinal has a $\theta$-extensional (even $\infty$-extensional) computable cover. Therefore, fix a noncomputable ordinal $\alpha$ and write $\alpha=\omega^{\theta} \cdot \beta+\rho$ with $\rho<\omega^{\theta}$. Since $\rho<\omega^{\theta}, \rho$ is computable and hence has a $\theta$-extensional cover. Since $\beta>\omega$ (because $\alpha$ is not computable), $\omega^{\theta} \cdot \beta$ has a $\theta$-extensional cover by Lemma 6 . Therefore, by Lemma $5, \alpha$ has a $\theta$-extensional computable cover. So Lemmas 5 and 6 imply Theorem 4.

To prove Lemma 5 , fix $\theta$-extensional computable covers $\mathfrak{A}_{0}$ and $\mathfrak{A}_{1}$ of $\mathcal{S}_{0}$ and $\mathcal{S}_{1}$ respectively. The objects in the $\theta$-extensional computable cover of $\mathcal{S}_{0}+\mathcal{S}_{1}$ have the form $\mathcal{A}_{i}^{0}+\mathcal{A}_{j}^{1}$ where $\mathcal{A}_{i}^{0} \in \mathfrak{A}_{0}$ and $\mathcal{A}_{j}^{1} \in \mathfrak{A}_{1}$, with the caveat that one of $\mathcal{A}_{i}^{0}$ or $\mathcal{A}_{j}^{1}$ is allowed to be empty. The injective maps from $\mathcal{A}_{i}^{0}+\mathcal{A}_{j}^{1}$ to $\mathcal{A}_{k}^{0}+\mathcal{A}_{\ell}^{1}$ are defined in the obvious way, and one checks that this cover is $\theta$-extensional.

The proof of Lemma 6 is notationally cumbersome, but the fundamental idea is that $\theta$-extensionality cannot distinguish between gaps in a linear order of length $\omega^{\theta} \cdot \gamma$ for varying nonzero values of $\gamma$. Each $\mathcal{A}_{i}$ in our $\theta$-extensional cover $\mathfrak{A}$ of $\omega^{\theta} \cdot \beta$, is a finite linear order of the form $1<2<\cdots<n$, for some $n$, together with an $n$-tuple $\left\langle\xi_{0}, \xi_{1}, \ldots, \xi_{n-1}\right\rangle$, called its label, in which each $\xi_{i} \in \omega^{\theta} \cdot 2$. If $\xi_{i}<\omega^{\theta}$, then $\xi$ indicates that the gap between $i$ and $i+1$ (or the gap to the left of 1 if $i=0$ ) in $\mathcal{A}_{i}$ should have length $\xi_{i}$. If $\xi_{i}=\omega^{\theta}+\rho$, then it indicates that this gap has length $\omega^{\theta} \cdot \gamma+\rho$ for some $\gamma \geq 1$. (Note that the labels are not formally part of $\mathcal{A}_{i}$. They are merely a denotation to help us keep track of which injective maps to include in $I_{i j}^{\mathfrak{A}}$.)

For each $n$ and each label $\left\langle\xi_{0}, \ldots, \xi_{n-1}\right\rangle$, we include an object $\mathcal{A}_{i}$ of length $n$ with this label in $\mathfrak{A}$. Let $\mathcal{A}_{i}$ have domain $\{1, \ldots, n\}$ with label $\left\langle\xi_{0}, \ldots, \xi_{n-1}\right\rangle$ and $\mathcal{A}_{j}$ have domain $\{1, \ldots, m\}$ with label $\left\langle\eta_{0}, \ldots, \eta_{m-1}\right\rangle$. We include an order 
preserving map $f:\{1, \ldots, n\} \rightarrow\{1, \ldots, m\}$ in $I_{i j}^{\mathfrak{A}}$ if and only if the labels match in the sense that $\xi_{0}=\eta_{0}+\cdots+\eta_{f(1)-1}$ and for all $0<k<n, \xi_{k}=$ $\eta_{f(k)}+\eta_{f(k)+1}+\cdots+\eta_{f(k+1)-1}$. It is straightforward to check that this process defines a computable cover of $\omega^{\theta} \cdot \beta$. To check that this cover is $\theta$-extensional takes longer and will not be presented here. The key fact is the following lemma, which can be established by induction on $\zeta$. Essentially it says that $\zeta$-extensional maps cannot distinguish one nonzero multiple of $\omega^{\zeta}$ from another, so that an object with (say) two elements $1<2$ and a gap labeled $\omega^{\zeta} \cdot \mu+\rho$ between them (where $\rho<\omega^{\zeta}$ ) can be used to cover two elements of the ordinal $\omega^{\theta} \cdot \beta$ by a map $\psi$, provided that $\psi(1)+\omega^{\zeta} \cdot \bar{\mu}+\rho=\psi(2)$ for some $\bar{\mu}$ which equals 0 iff $\mu=0$.

Lemma 7. Fix $\zeta \leq \theta$ and let $\psi: \mathcal{A}_{i} \rightarrow \omega^{\theta} \cdot \beta$ be an increasing map. Assume $\mathcal{A}_{i}$ has domain $\{1, \ldots, n\}$ and label $\left\langle\xi_{0}, \ldots, \xi_{n-1}\right\rangle$. Write each $\xi_{k}=\omega^{\zeta} \cdot \mu_{k}+\rho_{k}$ with $\rho_{k}<\omega^{\zeta}$. If there are ordinals $\bar{\mu}_{k}$, with $\bar{\mu}_{k}=0$ if and only if $\mu_{k}=0$, such that

$$
\psi(k)=\left(\omega^{\zeta} \cdot \bar{\mu}_{0}+\rho_{0}\right)+\left(\omega^{\zeta} \cdot \bar{\mu}_{1}+\rho_{1}\right)+\cdots+\left(\omega^{\zeta} \cdot \bar{\mu}_{k-1}+\rho_{k-1}\right),
$$

then $\psi$ is a $\zeta$-extensional map from $\mathcal{A}_{i}$ into $\omega^{\theta} \cdot \beta$.

This completes the proof sketch for Theorem 4. The full proof is quite technical and is omitted here. We believe that Theorem 4 ought to be a corollary to a more general result connecting effectiveness properties of the $\theta$ back-and-forth types of a structure with its being $\theta$-extensionally locally computable. We invite the interested reader to find the deeper connection that has thus far eluded us.

With Theorem 4, the general question of $\theta$-extensionality of ordinals $\alpha$ is now settled in almost all cases. When $\alpha<\omega_{1}^{C K}$ or $\theta<\omega_{1}^{C K}$, the answer is positive, by Proposition 1 and Theorem 4. When $\omega_{1}^{C K} \leq \alpha<\omega_{1}^{C K} \cdot \omega$, the answer is negative for every $\theta \geq \omega_{1}^{C K}$ by Proposition 2 and Theorem 3. When $\alpha \geq \omega_{1}^{C K} \cdot \omega$, the answer is negative for every $\theta>\omega_{1}^{C K}$ by Theorem 2. The only case remaining open is that in which $\theta=\omega_{1}^{C K}$ and $\alpha \geq \omega_{1}^{C K} \cdot \omega$.

\section{References}

1. C.J. Ash \& J.F. Knight; Computable Structures and the Hyperarithmetical Hierarchy (Amsterdam: Elsevier, 2000).

2. V.S. Harizanov; Pure computable model theory, Handbook of Recursive Mathematics, vol. 1 (Amsterdam: Elsevier, 1998), 3-114.

3. R.G. Miller; Locally computable structures, in Computation and Logic in the Real World - Third Conference on Computability in Europe, CiE 2007, eds. B. Cooper, B. Löwe, \& A. Sorbi, LNCS 4497 (Springer-Verlag: Berlin, 2007), 575-584.

4. R.G. Miller; Local computability and uncountable structures, to appear in the $A S L$ Lecture Notes in Logic volume Effective Mathematics of the Uncountable, eds. N. Greenberg, J.D. Hamkins, D.R. Hirschfeldt, \& R.G. Miller.

5. R.G. Miller \& D. Mulcahey; Perfect local computability and computable simulations, in Logic and Theory of Algorithms, Fourth Conference on Computability in Europe, CiE 2008, eds. A. Beckmann, C. Dimitracopoulos, \& B. Löwe, LNCS 5028 (Berlin: Springer-Verlag, 2008), 388-397.

6. R.I. Soare; Recursively Enumerable Sets and Degrees (New York: Springer, 1987). 